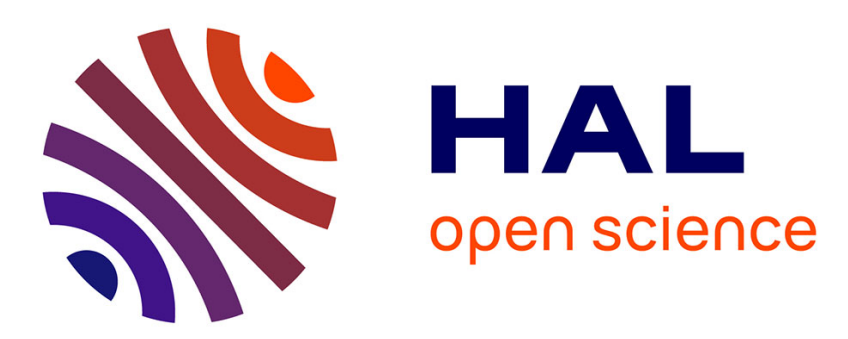

\title{
ON POLYMORPHISM FOR DISCRETE EVOLUTIONARY DYNAMICS DRIVEN EITHER BY SELECTION OR SEGREGATION DISTORTION
}

Thierry Huillet, Servet Martínez, Martin Möhle

\section{- To cite this version:}

Thierry Huillet, Servet Martínez, Martin Möhle. ON POLYMORPHISM FOR DISCRETE EVOLUTIONARY DYNAMICS DRIVEN EITHER BY SELECTION OR SEGREGATION DISTORTION. Journal of Applied and Computational Mathematics, 2016, 37 (2), pp.1352-1368. 10.1007/s40314016-0403-z . hal-01396515

\section{HAL Id: hal-01396515 \\ https://hal.science/hal-01396515}

Submitted on 14 Nov 2016

HAL is a multi-disciplinary open access archive for the deposit and dissemination of scientific research documents, whether they are published or not. The documents may come from teaching and research institutions in France or abroad, or from public or private research centers.
L'archive ouverte pluridisciplinaire HAL, est destinée au dépôt et à la diffusion de documents scientifiques de niveau recherche, publiés ou non, émanant des établissements d'enseignement et de recherche français ou étrangers, des laboratoires publics ou privés. 


\title{
ON POLYMORPHISM FOR DISCRETE EVOLUTIONARY DYNAMICS DRIVEN EITHER BY SELECTION OR SEGREGATION DISTORTION
}

\author{
THIERRY HUILLET ${ }^{1}$, SERVET MARTÍNEZ ${ }^{2}$, MARTIN MÖHLE $^{3}$
}

\begin{abstract}
We revisit some problems arising in the context of multiallelic discrete-time and deterministic evolutionary dynamics driven first by fitness differences and then by segregation distortion. In the model with fitness, we describe classes of fitness matrices exhibiting polymorphism. In the segregation case, still in search for conditions of polymorphism, we focus on a class of skew-symmetric matrices with a unique strictly positive kernel vector. Our main results reduce the study of these cases to the analysis of stochastic matrices.
\end{abstract}

Keywords: species frequencies dynamics, selection, segregation, polymorphism, stability, potential and stochastic matrices.

\section{INTRODUCTION}

We will first briefly revisit the basics of the deterministic dynamics arising in discrete-time asexual multiallelic evolutionary genetics driven only by fitness differences (or selection) and we will mainly consider the diploid case with $K$ alleles. In the diploid case, there is a deterministic updating dynamics of the full array of the genotype frequencies that involves the nonnegative symmetric fitness matrix $W \succeq \mathbf{0}$ (with $W=W^{\prime}, W^{\prime}$ the transpose of $W$ ) attached to the genotypes. When the Hardy-Weinberg law applies, it is sufficient to treat the induced marginal allelic frequencies dynamics. The updating dynamics on the simplex involves the mean fitness as a quadratic form in the current frequencies whereas marginal fitnesses are affine functions in these frequencies. The induced dynamics turns out to be gradient-like. We will also consider an alternative updating mechanism of allelic frequencies on the simplex, namely segregating distortion: here the fitness matrix $W$ is based on skew-symmetric matrices and the fitness landscape is flat. The induced relative frequencies dynamics is divergence-free like.

In the first case driven by viability selection and in the diploid context, the Fisher theorem, stating that the mean fitness increases as time passes by, holds true but, as a result of the fitness landscape being quadratic, a polymorphic equilibrium state will possibly emerge. Due to its major evolutionary interest, our subsequent concern is to identify examples of diploid dynamics leading to a unique polymorphic state $\mathbf{x}_{e q}$ on the simplex, either unstable or stable. We start with the unstable case and draw the attention on a class of fitness matrices leading to a unique unstable polymorphic equilibrium state: the class of potential matrices which includes the class of ultrametric matrices. For such potential fitness matrices, the mean fitness 
quadratic form is positive-definite. We will then consider a related class of fitness matrices with a mean fitness quadratic form which is negative-definite on a hyperplane of codimension one. For this class of matrices, there will also be a unique polymorphic equilibrium state for the diploid dynamics and it will be stable.

In the second case driven by segregation distortion, fitness is flat in the sense that its mean fitness quadratic form is a constant (the segregation ratio or fraction of $A_{k}$ gametes contributed by $A_{k} A_{l}$ individuals is $W_{k, l} \geq 0$ with $W_{k, l}+W_{l, k}=$ Const). Conditions under which its skew-symmetric part $\left(W-W^{\prime}\right) / 2$ has a strictly positive kernel will be addressed. If this is indeed the case, such models will also display a polymorphic equilibrium state and the flat fitness dynamics will spiral away from it to eventually reach the boundary of the state-space (as a simplex) in infinite time. In (Weissing and van Boven 2001), a population without sex-differentiation driven simultaneously by viability selection and segregation distortion is considered in Section 2, together with the adjunction of the effect of sex in Section 3. In this note, we shall study separately systems driven first by selection (in the absence of segregation distortion when segregation is Mendelian $W_{k, l}=W_{l, k}=$ Const $/ 2$ ) and then by segregation distortion (in the absence of selection) and we shall not consider the effect of sex.

Our main results will therefore characterize different types of nonnegative or skewsymmetric matrices (relevant for polymorphism) in terms of stochastic matrices. Theorem 1 of Section 2 characterizes the symmetric nonnegative fitness matrices for which $\mathbf{x}_{e q}$ is an isolated polymorphic stable state, in terms of stochastic matrices. In Theorem 2 of Section 4, we characterize skew-symmetric matrices having a strictly positive probability kernel $\mathbf{x}_{e q}$ also in terms of stochastic matrices. The associated dynamics with a flat fitness landscape will then display an isolated polymorphic state $\mathbf{x}_{e q}$, but it will be unstable.

\section{Single LOCUS: Diploid POPUlation With $K$ ALlELES}

2.1. Joint and marginal allelic dynamics (Bürger 2000, Ewens 2004). Consider $K$ alleles $A_{k}, k \in I_{K}:=\{1, \ldots, K\}$ attached at a single locus. Let $W=\left(W_{k, l}: k, l \in I_{K}\right)$ be some nonnegative fitness matrix. The coefficient $W_{k, l} \geq 0$ stands for the absolute fitness of the genotypes $A_{k} A_{l}$. Since $W_{k, l}$ is proportional to the probability of an $A_{k} A_{l}$ surviving to maturity, it is natural to assume that $W$ is symmetric.

Let $X=\left(x_{k, l}: k, l \in I_{K}\right)$ be the current frequency distribution at (integer) time $t$ of the genotypes $A_{k} A_{l}$, so $x_{k, l} \geq 0$ and $\sum_{k, l} x_{k, l}=1$. The joint evolutionary fitness dynamics in the diploid case is given by $X(t+1)=\mathbf{P}(X(t))$, with

$$
\mathbf{P}(X)_{k, l}=\frac{x_{k, l} W_{k, l}}{\omega(X)} \text { and } \omega(X)=\sum_{k, l} x_{k, l} W_{k, l} .
$$

The relative fitness of $A_{k} A_{l}$ is $W_{k, l} / \omega(X)$ and $\omega(X)$ is the mean fitness. The genotypic variance in absolute fitness is $\sigma^{2}(X)=\sum_{k, l=1}^{K} x_{k, l}\left(W_{k, l}-\omega(X)\right)^{2}$ and the diploid variance in relative fitness is $\bar{\sigma}^{2}(X)=\sigma^{2}(X) / \omega(X)^{2}$. Note that

$$
\Delta \omega(X)=\sum_{k, l} \Delta x_{k, l} W_{k, l}=\sum_{k, l} x_{k, l}\left(\frac{W_{k, l}^{2}}{\omega(X)}-W_{k, l}\right)=\omega(X) \bar{\sigma}^{2}(X) \geq 0
$$


with a relative rate of increase: $\Delta \omega(X) / \omega(X)=\bar{\sigma}^{2}(X)$. This expression vanishes only when $W_{k, l}$ is constant. This is the full diploid version of the Fisher theorem for asexuals; see Okasha 2008 for a deeper insight on the meaning of this theorem.

Assuming Hardy-Weinberg proportions, the frequency distribution at time $t$ of the genotypes $A_{k} A_{l}$ is given by: $x_{k, l}=x_{k} x_{l}$ where $x_{k}=\sum_{l} x_{k, l}$ is the marginal frequency of allele $A_{k}$ in the whole genotypic population. The frequency information is now contained in $\mathbf{x}=X \mathbf{1}^{1}$, where $\mathbf{1}^{\prime}:=(1, \ldots, 1)$ is the 1 -row vector of dimension $K . \mathbf{x}:=\left(x_{k}: k \in I_{K}\right)$ belongs to the $K$-simplex

$$
S_{K}=\left\{\mathbf{x}:=\left(x_{k}: k \in I_{K}\right) \in \mathbb{R}^{K}: \mathbf{x} \succeq \mathbf{0},|\mathbf{x}|=1\right\} .
$$

Here $|\mathbf{x}|:=\sum_{k=1}^{K} x_{k}$ and $\mathbf{x} \succeq \mathbf{0}$ means that all $x_{k} \geq 0, k=1, \ldots, K$. The elements of $S_{K}$ are called states. The mean fitness is given by the quadratic form: $\omega(\mathbf{x}):=$ $\sum_{k, l} x_{k} x_{l} W_{k, l}=\mathbf{x}^{\prime} W \mathbf{x}$. We now have $\sigma^{2}(\mathbf{x})=\sum_{k, l=1}^{K} x_{k} x_{l}\left(W_{k, l}-\omega(\mathbf{x})\right)^{2}$ and $\bar{\sigma}^{2}(\mathbf{x})=\sigma^{2}(\mathbf{x}) / \omega(\mathbf{x})^{2}$.

We will consider the update of the allelic marginal frequencies $\mathbf{x}$ themselves. Define the frequency-dependent marginal fitness of $A_{k}$ by $w_{k}(\mathbf{x})=(W \mathbf{x})_{k}:=\sum_{l} W_{k, l} x_{l}$. For the vector $\mathbf{x}$ denote by $D_{\mathbf{x}}:=\operatorname{diag}\left(x_{1}, \ldots, x_{K}\right)$, the associated diagonal matrix. The marginal mapping $\mathbf{p}: S_{K} \rightarrow S_{K}$ of the dynamics is given by:

$$
\mathbf{x}(t+1)=\mathbf{p}(\mathbf{x}(t)), \text { where } \mathbf{p}(\mathbf{x})=\frac{1}{\omega(\mathbf{x})} D_{\mathbf{x}} W \mathbf{x}=\frac{1}{\omega(\mathbf{x})} D_{W \mathbf{x}} \mathbf{x} .
$$

This dynamics involves a multiplicative interaction between $x_{k}$ and $(W \mathbf{x})_{k}$, the $k$ th entry of the image $W \mathbf{x}$ of $\mathbf{x}$ by $W$ and a normalization by the quadratic form $\omega(\mathbf{x})=\mathbf{x}^{\prime} W \mathbf{x}$. Iterating, the time- $t$ frequency distribution is:

$$
\mathbf{x}(t)=\mathbf{p}^{t}(\mathbf{x}(0))
$$

where $\mathbf{x}(0) \in S_{K}$ is some initial condition and $\mathbf{p}^{t}=\mathbf{p} \circ \ldots \circ \mathbf{p}, t$-times.

Remark. If $W_{k, l}=w_{k} w_{l}$, then (3) boils down to

$$
\mathbf{x}(t+1)=\mathbf{p}(\mathbf{x}(t)), \text { where } \mathbf{p}(\mathbf{x})=\frac{1}{w(\mathbf{x})} D_{\mathbf{w}} \mathbf{x}=\frac{1}{w(\mathbf{x})} D_{\mathbf{x}} \mathbf{w} .
$$

Here $\mathbf{w}=\left(w_{1}, \ldots, w_{K}\right)^{\prime}$ and $w(\mathbf{x})=\sum_{l} w_{l} x_{l}$ is linear. This yields the haploid asexual selection dynamics.

For an alternative representation of the dynamics (3) take $\Delta \mathbf{x}=\mathbf{p}(\mathbf{x})-\mathbf{x}$ and define the symmetric positive-definite matrix $G(\mathbf{x})=D_{\mathbf{x}}\left(I-\mathbf{1 x}^{\prime}\right)$ with entries:

$$
G(\mathbf{x})_{k, l}=x_{k}\left(\delta_{k, l}-x_{l}\right) .
$$

Let $V_{W}(\mathbf{x})=\frac{1}{2} \log \omega(\mathbf{x})$. Then, (3) may be recast as the gradient-like dynamics (Shashahani 1979):

$$
\Delta \mathbf{x}=\frac{1}{\omega(\mathbf{x})} G(\mathbf{x}) W \mathbf{x}=G(\mathbf{x}) \nabla V_{W}(\mathbf{x}),
$$

with $|\Delta \mathbf{x}|=\mathbf{1}^{\prime} \Delta \mathbf{x}=0$ as a result of $\mathbf{1}^{\prime} G(\mathbf{x})=\mathbf{0}^{\prime}$. Note that

$$
\nabla V_{W}(\mathbf{x})^{\prime} \Delta \mathbf{x}=\nabla V_{W}(\mathbf{x})^{\prime} G(\mathbf{x}) \nabla V_{W}(\mathbf{x}) \geq 0 .
$$

\footnotetext{
${ }^{1}$ Throughout, a boldface variable, say $\mathbf{x}$, will represent a column-vector and its transpose, say $\mathbf{x}^{\prime}$, will be a row-vector. And $\mathbf{x} \succ \mathbf{0}$ means all entries of $\mathbf{x}$ are positive.
} 
One can easily check that (3), as a replicator dynamics, can also be recast under the (non-linear) more conventional Fokker-Planck-like form $\mathbf{x}^{\prime}(t+1)=\mathbf{x}^{\prime}(t) P(\mathbf{x}(t))$, where $P(\mathbf{x})$ is the $\mathbf{x}$-dependent matrix with $(k, l)$-entry $P(\mathbf{x})_{k, l}=W_{k, l} x_{l} / \mathbf{x}^{\prime} W \mathbf{x} \in$ $(0,1)$. This matrix is not stricto sensu stochastic, but we note however that $\mathbf{x}^{\prime} P(\mathbf{x}) \mathbf{1}=1$ when $\mathbf{x} \in S_{K}$.

The mean fitness $\omega(\mathbf{x})$, as a Lyapunov function, $\Delta \omega(\mathbf{x}) \geq 0$, see (Atkinson et al. 1960 and Kingman 1961b). Its partial rate of increase due to frequency shifts only is $\delta \omega(\mathbf{x}):=\sum_{k} \Delta x_{k} w_{k}(\mathbf{x})$. It satisfies

$$
\frac{\delta \omega(\mathbf{x})}{\omega(\mathbf{x})}=\sum_{k} x_{k}\left(\frac{w_{k}(\mathbf{x})}{\omega(\mathbf{x})}-1\right)^{2}=\sum_{k} \frac{\left(\Delta x_{k}\right)^{2}}{x_{k}}=\frac{\bar{\sigma}_{A}^{2}(\mathbf{x})}{2},
$$

$\bar{\sigma}_{A}^{2}(\mathbf{x})$ being the allelic variance in relative fitness. See (Price 1972, Ewens 2004 and Castilloux and Lessard 1995) and the discussion of these results in (Bürger 2000).

2.2. Equilibria (diploid case). The mean fitness increase phenomenon occurs till the evolutionary dynamics reaches an equilibrium state. We wish to briefly discuss the questions relative to equilibria in the diploid case.

In contrast with the haploid case, in the diploid situation, the dynamics (3) can have more complex equilibrium points, satisfying $w_{k}\left(\mathbf{x}_{e q}\right)=w_{1}\left(\mathbf{x}_{e q}\right), k=2, \ldots, K$ and $\left|\mathbf{x}_{e q}\right|=1$. To avoid linear manifolds of equilibria, we first assume that all principal minors of $W$ are nonsingular. In particular $W_{k, k}>0$ for all $k$. In this case, from the Bézout theorem, the number of isolated equilibria is bounded above by the number $2^{K}-1$ of faces of $S_{K}$, see (Tallis 1966).

When $W=I$ is the identity matrix, there are $2^{K}-1$ equilibrium points (the barycenters of the $\left(\begin{array}{c}K \\ l+1\end{array}\right) l$-dimensional faces, $\left.l=0, . ., K-1\right)$, but only one polymorphic equilibrium which is the barycenter $\mathbf{x}_{B}$ of $S_{K}$. The barycenter has minimal mean fitness and it is unstable. The 0 -faces are stable fixed points whereas the barycenters of the $l$ faces with $l \in I_{K-2}$ are unstable saddle-points. The simplex $S_{K}$ can be partitioned into the attraction basins of the stable 0 -face states.

Similar conclusions can be drawn when we start with $W=\left(I-D_{\boldsymbol{\lambda}}\right)^{-1}$, where $\boldsymbol{\lambda}:=$ $\left(\lambda_{k}: k \in I_{K}\right)$ satisfies $\mathbf{0} \preceq \boldsymbol{\lambda} \prec \mathbf{1}$ (meaning $\left.0 \leq \lambda_{k}<1, \forall k\right)$. In this case again, there is only one unstable polymorphic equilibrium which is $\mathbf{x}_{e q}=(\mathbf{1}-\boldsymbol{\lambda}) /(K-|\boldsymbol{\lambda}|) \in S_{K}$. Again, all fixed points but the 0 -faces are unstable and the simplex $S_{K}$ can be partitioned into the attraction basins of these stable 0 -face monomorphic states: however, in contrast with the haploid case, the type of the survivor is not necessarily the one of the fittest (with largest $\lambda_{k}$ ); it will depend on the initial condition.

Due to its evolutionary interest, we would like now to discuss the stability of a polymorphic state. Under the above assumptions on the principal minors of $W$, a unique polymorphic equilibrium state exists if and only if

(i) there is a unique $\mathbf{z} \succ \mathbf{0}$ for which $W \mathbf{z}=\mathbf{1}$.

Under this condition, the unique equilibrium polymorphic state is

$$
\mathbf{x}_{e q}=\frac{\mathbf{z}}{|\mathbf{z}|} \text {. }
$$


Note that its mean fitness is given by $\omega\left(\mathbf{x}_{e q}\right)=1 /|\mathbf{z}|$.

Let us analyze the stability of $\mathbf{x}_{e q}$. Under $(i)$, by Cramer's rule, with $W_{(k)}$ a $K \times K$ matrix obtained from $W$ while substituting its $k$-th column by $\mathbf{1}$ (satisfying $\left|W_{(k)}\right|=(\operatorname{adj}(W) \mathbf{1})_{k}$, where $\operatorname{adj}(W)$ is the adjugate matrix of $W$, (see Nagylaki 1992, page 56), we have $|W|:=\operatorname{det}(W) \neq 0$ and

$$
z_{k}=\frac{\left|W_{(k)}\right|}{|W|}>0, k \in I_{K} .
$$

In (Kingman 1961a and 1961b), the condition to have stability of the polymorphic state $\mathbf{x}_{e q}=\mathbf{z} /|\mathbf{z}|$ was given. This occurs only when the following additional condition holds, (Lessard and Karlin 1982)

(ii) $W$ has exactly one strictly positive dominant eigenvalue and at least one strictly negative eigenvalue or else the sequence of the leading principal minors of $W$ alternates in sign, starting positive (Mandel 1959).

Under $(i)$ and $(i i), \mathbf{x}_{e q}$ is a stable equilibrium polymorphic state, and the stability is in the sense that it is a local maximum of the mean fitness $\omega(\mathbf{x})$, so a perturbation of it will result in a mean fitness decrease. The condition for $\mathbf{x}_{e q}$ to be an isolated global maximum of the fitness landscape $\omega(\mathbf{x})$ is that for all $\mathbf{x} \in S_{K}$ with $\mathbf{x} \neq \mathbf{x}_{e q}$ we have $\mathbf{x}^{\prime} W \mathbf{x}<\mathbf{x}_{e q}^{\prime} W \mathbf{x}_{e q}$. A direct computation shows (Kingman 1961a)

$$
\forall \mathbf{y} \neq \mathbf{0}, \mathbf{y} \perp \mathbf{1}: \mathbf{y}^{\prime} W \mathbf{y}<0 .
$$

In this case, starting from any initial condition in the interior of $S_{K}$, all trajectories will be attracted by $\mathbf{x}_{e q}$. In Theorem 1 below, we characterize the symmetric nonnegative fitness matrices for which $\mathbf{x}_{e q}$ is an isolated polymorphic stable state, in terms of stochastic matrices.

When there is no such unique globally stable polymorphic equilibrium, all trajectories will still converge but perhaps to a local equilibrium state where some alleles have gone extinct (Lyubich et al. 1980). Which allele and how many alleles are concerned seems to be an unsolved problem in its full generality.

We note that when the leading principal minors of $W$ are all positive, $\mathbf{x}_{e q}$ is unstable and the quadratic form $\mathbf{x}^{\prime} W \mathbf{x}$ is positive-definite.

In the sequel we will denote by $J:=\mathbf{1 1}^{\prime}$ the flat $K \times K$ matrix whose entries are all 1.

Theorem 1. Let $W$ be a nonnegative symmetric matrix and suppose there exists $\mathbf{z} \succ \mathbf{0}$ such that $W \mathbf{z}=\mathbf{1}$. Then, $W$ admits the representation $W=P D_{\mathbf{z}}^{-1}$, where $P$ is a stochastic irreducible matrix obeying detailed balance with respect to $\mathbf{z}^{\prime}$, and conversely.

Assume $W$ satisfies the above conditions. Then,

(i) $W$ is positive-definite if and only if $P$ has a positive spectrum;

(ii) The matrix $W$ satisfies

$$
\sup \left\{\mathbf{y}^{\prime} W \mathbf{y}: \mathbf{y} \perp \mathbf{1}, \mathbf{y} \neq \mathbf{0}\right\}<0,
$$

if and only if $P$ has 1 as unique positive eigenvalue, all the other ones being negative. Therefore, this is a sufficient condition in order that $\mathbf{x}_{e q}$ be a polymorphic stable state for $W$; 
(iii) If $P$ is a stochastic matrix that satisfies detailed balance with respect to some $\mathbf{z} \succ \mathbf{0}$ and $\lambda>\lambda_{0}$, where $\lambda_{0}=\min (\operatorname{spectrum}(P))$ then the stochastic matrix $P_{\lambda}=$ $\left(1+\lambda_{0}\right)^{-1}\left(P+\lambda_{0} I\right)$ satisfies detailed balance with respect to $\mathbf{z}$ and it has a positive spectrum. Furthermore, every stochastic matrix that satisfies detailed balance and has positive spectrum can be constructed in this way;

(iv) Assume $W$ is a positive-definite nonnegative symmetric matrix for which there exists $\mathbf{z} \succ \mathbf{0}$ with $W \mathbf{z}=\mathbf{1}$. For $\xi>\max _{i, j} W_{i, j}$, define

$$
\bar{W}=\xi J-W .
$$

Then $\bar{W}$ is a nonnegative symmetric matrix for which $\mathbf{z} /|\mathbf{z}|$ is a polymorphic stable state.

Proof: Assume that $W \geq 0$ is a symmetric matrix and $W \mathbf{z}=\mathbf{1}$ for some $\mathbf{z} \succ \mathbf{0}$. Then $W D_{\mathbf{z}} \mathbf{1}=\mathbf{1}$ and so $P=W D_{\mathbf{z}}$ is a stochastic matrix; clearly, $W^{\prime}=W$ is equivalent to $P^{\prime}=D_{\mathbf{z}} P D_{\mathbf{z}}^{-1}$. Since $P^{\prime} \mathbf{z}=\mathbf{z}, \mathbf{z}$ is proportional to the invariant probability measure of $P$. The reciprocal follows immediately, because detailed balance for the stochastic matrix $P$ means $P^{\prime}=D_{\mathbf{z}} P D_{\mathbf{z}}^{-1}$ and so $W=P D_{\mathbf{z}}^{-1}$ is a nonnegative symmetric matrix for which $W \mathbf{z}=\mathbf{1}$.

Let us now prove condition $(i)$, namely that $W$ has a positive spectrum if and only if $P$ also does. Since $P$ obeys detailed balance we have that $Q=D_{\mathbf{z}}^{1 / 2} P D_{\mathbf{z}}^{-1 / 2}$ is symmetric with the same spectrum as $P$. We have $W=P D_{\mathbf{z}}^{-1}=D_{\mathbf{z}}^{-1 / 2} Q D_{\mathbf{z}}^{-1 / 2}$. Hence, $W$ is positive-definite if and only if $Q$ is positive-definite, and this happens if and only if $Q$ has a positive spectrum by an argument given in (Kingman 1961b). And so if and only if $P$ has a positive spectrum.

Let us show (ii) which is a rephrasing of (Kingman 1961a, section 3). From $P \mathbf{1}=\mathbf{1}$, we get that the matrix $Q$ has eigenvalue 1 with eigenvector $D_{\mathbf{z}}^{1 / 2} \mathbf{1}$. Then, all other eigenvalues of $P$ are negative if and only if all other eigenvalues of $Q$ are negative and since $Q$ is symmetric, this condition is equivalent to $\sup \left\{\mathbf{y}^{\prime} Q \mathbf{y}: \mathbf{y} \perp D_{\mathbf{z}}^{1 / 2} \mathbf{1}, \mathbf{y}\right.$ $\neq \mathbf{0}\}<0$. Substituting $\mathbf{y}$ for $D_{\mathbf{z}}^{1 / 2} \mathbf{y}$, and because $\mathbf{z}>\mathbf{0}$, this is equivalently

$$
\left\{\mathbf{y}^{\prime} D_{\mathbf{z}}^{-1 / 2} Q D_{\mathbf{z}}^{-1 / 2} \mathbf{y}: \mathbf{y} \perp \mathbf{1}, \mathbf{y} \neq \mathbf{0}\right\}<0 .
$$

Recalling $Q=D_{\mathbf{z}}^{1 / 2} P D_{\mathbf{z}}^{-1 / 2}$, this is also $\mathbf{y}^{\prime} P D_{\mathbf{z}}^{-1} \mathbf{y}: \mathbf{y} \perp \mathbf{1}, \mathbf{y} \neq \mathbf{0}<0$. Which is

$$
\sup \left\{\mathbf{y}^{\prime} W \mathbf{y}: \mathbf{y} \perp \mathbf{1}, \mathbf{y} \neq \mathbf{0}\right\}<0,
$$

because $W=P D_{\mathbf{z}}^{-1}$. This is the condition (7) in order that $\mathbf{x}_{e q}$ be a polymorphic stable state for $W$.

Part (iii) is a direct computation.

Let us prove part $(i v)$. Let $W$ be a positive-definite nonnegative symmetric matrix and $\mathbf{z} \succ \mathbf{0}$ such that $W \mathbf{z}=\mathbf{1}$. Then $\bar{W}=\xi J-W$ with $\xi>\max _{i j} W_{i, j}$ satisfies $\bar{W} \succeq \mathbf{0}$ and $\bar{W} \mathbf{z}=(\xi|\mathbf{z}|-1) \mathbf{1}$. Note that $\xi>\max _{i, j} W_{i, j}$ and $W \mathbf{z}=\mathbf{1}$ imply $\xi|\mathbf{z}|>1$. Moreover, since $W$ is positive-definite, we get that for all vector $\mathbf{y} \perp \mathbf{1}$ we have $\mathbf{y}^{\prime} \bar{W} \mathbf{y}=\mathbf{y}^{\prime}\left(\xi \mathbf{1 1} \mathbf{1}^{\prime}-W\right) \mathbf{y}=-\mathbf{y}^{\prime} W \mathbf{y}<0$, so the stability condition (7) is satisfied. 
We note that in (Kingman 1961a, section 3), the symmetric matrix $Q$ was used for the study of stability.

In the case when $W$ is a symmetric bi-stochastic matrix we have $W \mathbf{1}=\mathbf{1}$, and since $D_{1}=I$, the identity matrix, we get $P=W$ in the representation given in the theorem. Let $S$ be a symmetric permutation matrix so $S=S^{\prime}=S^{-1}$ and it is bistochastic. Let $\mathbf{y} \succ \mathbf{0}$ and consider $W=D_{\mathbf{y}}^{-1} S D_{\mathbf{y}}^{-1}$. Putting $\mathbf{z}=W^{-1} \mathbf{1}=D_{\mathbf{y}} S \mathbf{y}$, we have $\mathbf{z} \succ \mathbf{0}$ and $W \mathbf{z}=\mathbf{1}$. In this case $D_{\mathbf{z}}=D_{\mathbf{y}} S D_{\mathbf{y}}$ and so $P=W D_{\mathbf{z}}$ is the identity matrix, which is stochastic and every positive vector satisfies the detailed balance with respect to it, in particular $\mathbf{z}$.

2.3. Potential matrices. Let us introduce potential matrices associated to strictly substochastic matrices. Let $\Lambda$ be a strictly substochastic matrix, so $\Lambda \succeq \mathbf{0}$ and $\mathbf{0} \prec \Lambda \mathbf{1}:=\mathbf{q} \prec \mathbf{1}$. We will also assume that $\Lambda$ is irreducible. Then, $W=(I-\Lambda)^{-1}$ exists and it is the potential matrix associated to $\Lambda$ (else the resolvent matrix $R_{\Lambda}(z)=(z I-\Lambda)^{-1}$, evaluated at $\left.z=1\right)$. We have $W \succ \mathbf{0}$ and $W$ is obviously nonsingular. For any $\lambda>0$, the matrix $W=\lambda^{-1}(I-\Lambda)^{-1}$ will also be called a potential matrix, parameterized by $\lambda$.

The equilibrium potential (vector) of a potential matrix $W$ is by definition $\mathbf{z}=$ $W^{-1} \mathbf{1}$. Hence, for all $\lambda>0, \lambda \mathbf{1} \succ \mathbf{z}=\lambda(I-\Lambda) \mathbf{1}=\lambda(\mathbf{1}-\mathbf{q}) \succ \mathbf{0}$ is satisfied. We have: $|\mathbf{z}|=\lambda(K-|\mathbf{q}|)$ and

$$
\mathbf{z}=\lambda(I-\Lambda) \mathbf{1}=\lambda(\mathbf{1}-\mathbf{q}) \succ \mathbf{0} .
$$

When $\Lambda$ is symmetric it has real eigenvalues and all them are strictly smaller than 1 . Hence, the symmetric potential matrix $W$ is positive-definite because when solving $W \mathbf{u}=\xi \mathbf{u}$ we get $\Lambda \mathbf{u}=(1-1 /(\xi \lambda)) \mathbf{u}$ and we deduce $\xi>0$. The potential matrices $W$ are row diagonal dominant in the sense that $W_{k, k}=\max _{l} W_{k, l}$.

We can make the construction done in Theorem 1 part $(i v)$, that is matrices of type (8). Let $W=\lambda^{-1}(I-\Lambda)^{-1}$ be a symmetric potential matrix. Then $\mathbf{z}=\lambda(\mathbf{1}-\mathbf{q}) \succ \mathbf{0}$ satisfies $W \mathbf{z}=\mathbf{1}$. For $\xi>\max _{k, l} W_{k, l}=\max _{k} W_{k, k}$, define the matrix $\bar{W}$ as in (8): $\bar{W}=\xi J-W$. Then, $\bar{W} \succ \mathbf{0}$ and $\bar{W} \mathbf{z}=(\xi-|\mathbf{z}|) \mathbf{1}$. We note that $\xi>\max _{k, l} W_{k, l}$ and $W \mathbf{z}=\mathbf{1}$ implies that $\xi-|\mathbf{z}|>0$, and so $\overline{\mathbf{z}}=\mathbf{z}(\xi-|\mathbf{z}|) \mathbf{z}$ is an equilibrium potential of $\bar{W}$. From Theorem 1 parts (ii) and (iv), we get that $\mathbf{x}_{e q}$ is a stable polymorphic state for $\bar{W}$ and $\bar{\omega}\left(\mathbf{x}_{e q}\right)=(\xi-|\mathbf{z}|) /|\mathbf{z}|$ is the mean fitness at equilibrium.

2.4. Ultrametric matrices. Let us introduce ultrametric matrices, which in (Martínez et al. 1994) were proven to be potential matrices. The symmetric nonnegative matrix $W=\left(W_{i, j}: i, j \in I_{K}\right)$ is called an ultrametric matrix if it satisfies

$$
\forall j, k, l \in I_{K}: W_{k, l} \geq \min \left\{W_{k, j}, W_{j, l}\right\} .
$$

This condition implies $W_{k, k} \geq \max _{l \neq k}\left\{W_{k, l}\right\}, \forall k \in I_{K}$. If $W$ satisfies this last condition but in a strict way, that is $W_{k, k}>\max _{l \neq k}\left\{W_{k, l}\right\} \forall k \in I_{k}$, then $W$ is called a strictly ultrametric matrix. An ultrametric matrix is nonsingular if no row and no column is equal to 0 , and no two rows (or columns) are equal. So, a strictly ultrametric matrix is nonsingular. If $W$ is a nonsingular ultrametric matrix, it is a potential matrix having a nonnegative equilibrium potential $\mathbf{z}=W^{-1} \mathbf{1}$ and if $W$ is a strictly ultrametric matrix then $\mathbf{z} \succ \mathbf{0}$. 
When $K>1$, by a permutation of indices in $I_{K}$, an ultrametric matrix can always be written in a nested block form (NBF), meaning that we can write it in the form,

$$
W=\left(\begin{array}{cc}
W^{1} & \alpha_{W} \mathbf{1}_{I^{1}} \mathbf{1}_{I^{2}}^{\prime} \\
\alpha_{W} \mathbf{1}_{I^{2}} \mathbf{1}_{I^{1}}^{\prime} & W^{2}
\end{array}\right)
$$

where $I_{K}=I^{1} \cup I^{2}, \alpha_{W}=\min _{i, j} W_{i j}$ and $W^{1}$ and $W^{2}$ are ultrametric matrices in $\mathrm{NBF}$. Here, the vector $\mathbf{1}_{I^{l}}$ denotes the 1 -constant vector of dimension $\left|I^{l}\right|, l=1,2$. Then,

$$
W=\alpha_{W} \mathbf{1 1 ^ { \prime }}+\left(\begin{array}{cc}
\widehat{W}^{1} & 0 \\
0 & \widehat{W}^{2}
\end{array}\right),
$$

where $\widehat{W}^{i}:=W^{i}-\alpha_{W} \mathbf{1 1}^{\prime}, i=1,2$, are again ultrametric.matrices. If $\left|I^{1}\right|>1$, by permuting the indices in $I^{1}$ if necessary, we now have

$$
\widehat{W}^{1}=\alpha_{\widehat{W}^{1}} \mathbf{1}_{I^{1}} \mathbf{1}_{I^{1}}^{\prime}+\left(\begin{array}{cc}
\widehat{W}^{3} & 0 \\
0 & \widehat{W^{4}}
\end{array}\right)
$$

and so, with $\widehat{1}_{I^{1}}$ the vector of dimension $|I|$ whose coordinates in $I^{1}$ are equal to 1 and those in $I \backslash I^{1}$ are equal to 0 , we get

$$
W=\alpha_{W} \mathbf{1} \mathbf{1}^{\prime}+\alpha_{\widehat{W}^{1}} \widehat{\mathbf{1}}_{I^{1}} \widehat{\mathbf{1}}_{I^{1}}^{\prime}+\left(\begin{array}{ccc}
\widehat{W}^{3} & 0 & 0 \\
0 & \widehat{W}^{4} & 0 \\
0 & 0 & \widehat{W}^{2}
\end{array}\right) .
$$

If (extending what we have already used for $l=1$ to all $l$ ) we define $\widehat{1}_{I^{l}}$ to be the vector of dimension $|I|$ whose coordinates in $I^{l}$ are equal to 1 and those in $I \backslash I^{l}$ are equal to 0 , iterating the above construction with $K>1$, we can partition $W$ while generating a sequence of partition matrices $\widehat{W}^{1}, \ldots, \widehat{W}^{r}$ with $r \geq K-2$, till we end in a diagonal matrix $D$. But only $K-2$ of these matrices are not $1 \times 1$ matrices and so can be subsequently partitioned. Let us denote this subsequence by $\widehat{W}^{l_{1}}, \ldots, \widehat{W}^{l_{K-2}}$. Starting from $W$, and ending with the diagonal matrix, we obtain

$$
W=\alpha_{W} \mathbf{1} \mathbf{1}^{\prime}+\sum_{r=1}^{K-2} \alpha_{\widehat{W}^{l_{r}}} \widehat{\mathbf{1}}_{I^{l_{r}}} \widehat{\mathbf{1}}_{I^{l_{r}}}^{\prime}+D
$$

Note that all the diagonal elements of the terminal matrix $D$ are strictly positive if $W$ is strictly ultrametric.

Stated differently, the above construction gives a real sequence $s_{l} \geq 0$ and a sequence of vectors $\mathbf{u}_{l}$ for $l=1, \ldots, 2 K-1$ with entries in $\{0,1\}$ such that (see Nabben and Varga 1994)

$$
W=\sum_{l=1}^{2 K-1} s_{l} \mathbf{u}_{l} \mathbf{u}_{l}^{\prime} .
$$

For strictly ultrametric matrix all the $1 \times 1$ matrices corresponding to the $K$ diagonal terms should be strictly positive, so there should be at least $K$ terms $s_{l}>0$. 
If $W$ is an ultrametric matrix, then the associated matrix $\bar{W}$ defined in (8), satisfies the following ultrametric property,

$$
\forall j, k, l: \bar{W}_{k, l} \leq \max \left\{\bar{W}_{k, j}, \bar{W}_{j, l}\right\},
$$

and if $W$ is strictly ultrametric then we have a strict fitness domination of the heterozygotes $A_{k} A_{l}$ over the homozygotes,

$$
\forall k: \bar{W}_{k, k}<\min _{l \neq k}\left\{\bar{W}_{k, l}\right\} .
$$

Then, $\bar{W}$ will display a stable polymorphic equilibrium state $\mathbf{x}_{e q}$.

Remark. The strictly ultrametric conditions (12) and (13) should be compared with the so-called 'triangle inequality' conditions, which are necessary for stable polymorphism, as first pointed out in (Lewontin et al. 1978). They read:

(i) $\quad \forall k \neq l \exists j, k \neq j \neq l$ with: $\bar{W}_{k, l}<\bar{W}_{k, j}+\bar{W}_{l, j}$;

(ii) $\quad \forall k \neq l: \bar{W}_{k, l}>\frac{1}{2}\left(\bar{W}_{k, k}+\bar{W}_{l, l}\right)$.

It is clear that the matrices $\bar{W}$ of type (8) derived from ultrametric matrices $W$ will satisfy these conditions.

Assume $K \geq 2$ and $W$ is a strictly ultrametric matrix, then

$$
\max \left\{W_{i j}: i, j \in I_{K}\right\}<\sum_{l=1}^{2 K-1} s_{l} .
$$

We can construct $\bar{W}=\xi J-W$ in (8) with $\xi=\sum_{l=1}^{2 K-1} s_{l}$ and so

$$
\bar{W}=s_{1} J+V \text { with } V:=\sum_{l=2}^{2 K-1} s_{l}\left(J-\mathbf{u}_{l} \mathbf{u}_{l}^{\prime}\right) .
$$

So, $V$ is of type (8) as well, then there is a $\mathbf{z}_{V} \succ \mathbf{0}$ such that $V \mathbf{z}_{V}=\mathbf{1}$. Thus $\bar{W} \mathbf{z}_{V}=\left(s_{1} J+V\right) \mathbf{z}_{V}=\left(s_{1}\left|\mathbf{z}_{V}\right|+1\right) \mathbf{1}$ showing that, with $\overline{\mathbf{z}}=\mathbf{z}_{V} /\left(s_{1}\left|\mathbf{z}_{V}\right|+1\right)$, $\bar{W} \overline{\mathbf{z}}=\left(s_{1} J+V\right) \overline{\mathbf{z}}=\mathbf{1}$.

In the case $s_{l}=1, \forall l$, any positive matrix of the form $\bar{W}=\gamma J+V$ with $\gamma>0$ will admit a stable polymorphic equilibrium state and the equilibrium mean fitness for $\mathbf{x}_{e q}=\mathbf{z}_{V} /\left|\mathbf{z}_{V}\right|$ is $\mathbf{x}_{e q}^{\prime} \bar{W} \mathbf{x}_{e q}=\left(\gamma\left|\mathbf{z}_{V}\right|+1\right) /\left|\mathbf{z}_{V}\right|$.

2.5. Examples. (i) Let $K=2, s>-1, h>0$ and $s h>-1$. The positive matrix

$$
W=\left(\begin{array}{cc}
1+s & 1+s h \\
1+s h & 1
\end{array}\right)
$$

defines the fitness matrix with selection parameter $s$ and dominance $h$. Here $\mathbf{x}_{e q}^{\prime}=$ $(h /(2 h-1) ;(h-1) /(2 h-1))$. The matrix $W$ is strictly ultrametric if and only if $s<0$ and $h>1$, and in this case the equilibrium state is unstable. The matrix $W$ is of type (8) if and only if $s>0$ and $h>1$, and in this case the equilibrium state is stable.

(ii) Let $K=4$, that is four alleles, with $W=\sum_{l=1}^{7} s_{l} \mathbf{u}_{l} \mathbf{u}_{l}^{\prime}$, being $s_{l}=1$ for $l=1, . ., 7, \mathbf{u}_{1}=(1,1,1,1), \mathbf{u}_{2}=(1,0,0,0), \mathbf{u}_{3}=(0,1,1,1), \mathbf{u}_{4}=(0,1,1,0), \mathbf{u}_{5}=$ 
$(0,0,0,1), \mathbf{u}_{6}=(0,1,0,0), \mathbf{u}_{7}=(0,0,1,0)$ and $\xi=7$. We find $\bar{W}=\xi J-W=J+V$ with:

$$
V=\left(\begin{array}{llll}
2 & 1 & 1 & 1 \\
1 & 4 & 3 & 2 \\
1 & 3 & 4 & 2 \\
1 & 2 & 2 & 3
\end{array}\right),
$$

which is a symmetric matrix of type (8). For this example, $\mathbf{z}_{V}^{\prime}=\frac{1}{150}(27,1,1,3)$ and $\mathbf{x}_{e q}^{\prime}=\frac{1}{32}(27,1,1,3)$ and the equilibrium mean fitness is $\mathbf{x}_{e q}^{\prime} \bar{W} \mathbf{x}_{e q}=\left(\left|\mathbf{z}_{V}\right|+\right.$ $1) /\left|\mathbf{z}_{V}\right|=91 / 66$.

\section{Flat fitness and segregation distortion (Weissing and van Boven 2001)}

We now address the flat fitness model. Let $A$ be a real skew-symmetric matrix, so obeying

$$
A^{\prime}=-A \text {. }
$$

Let $\sigma, \lambda>0$. We wish here to consider evolutionary dynamics of the form (3) but now when $W$ is of the form $W=\lambda^{-1}(J+\sigma A) \succeq \mathbf{0}$ when $A^{\prime}=-A$ and such that $\left|A_{k, l}\right| \leq 1 / \sigma$. The mean fitness function $\omega(\mathbf{x})$ appearing in (3) is a constant $\omega(\mathbf{x})=\mathbf{x}^{\prime} W \mathbf{x}=\lambda^{-1}$, and in this sense the fitness matrix $W$ is called flat. Because $W_{k, l}+W_{l, k}=2 \lambda^{-1}$, these models correspond to constant-sum games in which each pair of two players has opposed interest or to evolution under the effect of segregation distortion in population genetics; See (Karlin 1984, Hofbauer and Sigmund 1998, Weissing and van Boven 2001). An interesting sub-family of such models is when $\sigma \in(0,1]$ and $A_{k, l} \in\{-1,0,1\}$. Some authors recently and interestingly considered the interaction between selection differences and segregation distortion effects but we shall not run into this problem (Weissing and van Boven 2001; Sanyal and Sarkar 2013).

The dynamics (3) for this particular form of $W$ boils down to

$$
\mathbf{x}(t+1)=\mathbf{p}(\mathbf{x}(t)), \text { where } \mathbf{p}(\mathbf{x})=\frac{1}{\omega(\mathbf{x})} D_{\mathbf{x}} W \mathbf{x}=\mathbf{x}+\sigma D_{\mathbf{x}} A \mathbf{x},
$$

independent of $\lambda$. With $\Delta x_{k}(t)=x_{k}(t+1)-x_{k}(t)$, with $H(\mathbf{x}):=\sum_{k=1}^{K} x_{k}^{2} / 2$ the simple energy quadratic form, we have

$$
\frac{\Delta x_{k}(t)}{x_{k}(t)}=\frac{(W \mathbf{x})_{k}-1}{\omega(\mathbf{x})}=\sigma(A \nabla H(\mathbf{x}))_{k}, k=1, \ldots, K,
$$

with $\sigma \nabla \cdot(A \nabla H(\mathbf{x}))=0$ : the relative allelic frequencies obey a divergence-free dynamics.

Such $\mathbf{p}(\cdot)$ are called quadratic stochastic or Volterra operators, (Ganikhodzhaev et al. 2011). When $\sigma=1 /\left(\max A_{k, l}+c\right)$ with $c \geq 0$ some constant, it can be checked that the $K$ extremal points (0-faces) of $S_{K}$ are fixed points of (14). 
A key problem we shall face in the next Section is to determine those skewsymmetric matrices $A$ for which $A \mathbf{z}=\mathbf{0}$ for some $\mathbf{z} \succ \mathbf{0}$. Indeed, when such a $\mathbf{z}$ exists, with $\lambda=|\mathbf{z}|$, the new $W=\lambda^{-1}(J+\sigma A)$ obeys $W \mathbf{z}=\mathbf{1}$, with $\mathbf{z}$ an 'equilibrium potential' of $W ; \mathbf{x}_{e q}:=\mathbf{z} /|\mathbf{z}|$ will be a polymorphic equilibrium state of the dynamics (14) governed by the flat fitness matrix $W$.

Note that since $\omega(\mathbf{x})$ is constant, it is not a Lyapunov function. Define the partial rate of increase of the mean fitness due to frequency shifts as

$$
\delta \omega(\mathbf{x}):=\sum_{k} \Delta x_{k} w_{k}(\mathbf{x}), \text { where } w_{k}(\mathbf{x})=(W \mathbf{x})_{k} .
$$

Observing $\mathbf{x}^{\prime} A \mathbf{x}=\sum_{k} x_{k}(A \mathbf{x})_{k}=0$, it is satisfied

$$
\begin{aligned}
& \frac{\delta \omega(\mathbf{x})}{\omega(\mathbf{x})}=\delta \omega(\mathbf{x})=\left|\mathbf{z}_{0}\right| \sum_{k} \Delta x_{k}(W \mathbf{x})_{k}=\left|\mathbf{z}_{0}\right| \sum_{k} \Delta x_{k}\left(1+(A \mathbf{x})_{k}\right) \\
= & \left|\mathbf{z}_{0}\right| \sum_{k} x_{k}(A \mathbf{x})_{k}\left(\frac{1}{\left|\mathbf{z}_{0}\right|}+(A \mathbf{x})_{k}\right)=\left|\mathbf{z}_{0}\right| \sum_{k} x_{k}(A \mathbf{x})_{k}^{2}=\frac{\bar{\sigma}_{A}^{2}(\mathbf{x})}{2}>0,
\end{aligned}
$$

where $\bar{\sigma}_{A}^{2}(\mathbf{x})$ is the allelic variance in relative fitness (Price 1972).

Consider the dynamics $\mathbf{p}(\mathbf{x})=\frac{1}{\omega(\mathbf{x})} D_{\mathbf{x}} W \mathbf{x}=\frac{1}{\omega(\mathbf{x})} D_{W \mathbf{x}} \mathbf{x}$, where $W=J+A \succeq \mathbf{0}$, with $A$ skew-symmetric, $\left|A_{k, l}\right| \leq 1$ and such that there is a $\mathbf{z} \succ \mathbf{0}$ for which $A \mathbf{z}=\mathbf{0}$. So $\mathbf{p}(\mathbf{x})=\mathbf{x}+D_{\mathbf{x}} A \mathbf{x}$. Consider $V(\mathbf{x})=\prod_{k=1}^{K} x_{k}^{z_{k}}$, where $\mathbf{x} \in S_{K}$. Then $V$ is maximum at $\mathbf{x}_{e q}=\mathbf{z} /|\mathbf{z}|$ with $V\left(\mathbf{x}_{e q}\right)=|\mathbf{z}|^{|\mathbf{z}|} \prod_{k=1}^{K} z_{k}^{z_{k}}$. Clearly, in view of $(W x)_{k} \geq 0$ and $\mathbf{x}^{\prime} W \mathbf{x}=1$,

$$
V(\mathbf{p}(\mathbf{x}))=V(\mathbf{x}) \prod_{k=1}^{K}(W x)_{k}^{z_{k}}=V(\mathbf{x}) \prod_{k=1}^{K}\left(1+(A x)_{k}\right)^{z_{k}}<V(\mathbf{x}),
$$

the latter inequality following from the information inequality (Bapat and Raghavan 1997 , p. 80), the concavity of the function $\log$ and the Jensen's inequality.

This shows (Liberman 1991 and Ganikhodzhaev et al. 2011), that $\mathbf{x}_{e q}$ in the interior of $S_{K}$ is a repeller and that the trajectory of $\mathbf{x}$ spirals away $\mathbf{x}_{e q}$ towards the boundary of $S_{K}$, without ever reaching it in finite time (a quasi-fixation type event as defined in Kimura 1954).

3.1. Examples. (i) Let $A$ be a $K \times K$ skew-symmetric matrix. Let us show that for $K$ odd or even, the conditions under which there is a positive state $\mathbf{x}$ for which $A \mathbf{x}=\mathbf{0}$, is amenable to the sign structure of some determinants by Cramer's rule.

- The odd case. Let $K$ be odd. Suppose $A$ has rank $K-1$. So 0 is an algebraically and geometrically simple eigenvalue of $A$. We want to check under what conditions $A \mathbf{x}=\mathbf{0}$ for some unique $\mathbf{x} \succ \mathbf{0}$ with $|\mathbf{x}|=1$. We can decompose $A$ as follows, where $\overline{\mathbf{a}}$ is a size $K-1$ column vector and $\operatorname{rank}(\bar{A})=K-1$,:

$$
A=\left(\begin{array}{cc}
\bar{A} & \overline{\mathbf{a}} \\
-\overline{\mathbf{a}}^{\prime} & 0
\end{array}\right)
$$

If $A \mathbf{z}=\mathbf{0}$ with $\mathbf{z} \succ \mathbf{0}$, and $\mathbf{x}:=\mathbf{z} /|\mathbf{z}|$, we have $A \mathbf{x}=\mathbf{0}$ for some $\mathbf{x} \succ \mathbf{0}$ with $|\mathbf{x}|=1$. Since $\operatorname{rank}(\bar{A})=\operatorname{rank}(A)=K-1$, there exists a vector $\overline{\mathbf{b}}$ of size $K-1$ such that 
$\left(\begin{array}{c}\bar{A} \\ -\overline{\mathbf{a}}^{\prime}\end{array}\right) \overline{\mathbf{b}}=\left(\begin{array}{c}\overline{\mathbf{a}} \\ 0\end{array}\right)$. Then $\overline{\mathbf{b}}$ is uniquely given by inversion of $\bar{A} \overline{\mathbf{b}}=\overline{\mathbf{a}}$. We have $\overline{\mathbf{a}}^{\prime} \overline{\mathbf{b}}=0$ because $\overline{\mathbf{b}}^{\prime} \overline{\mathbf{a}}=\overline{\mathbf{b}}^{\prime} \bar{A} \overline{\mathbf{b}}=0$, in view of $\bar{A}$ being skew-symmetric. The vector $\mathbf{b}:=\left(\begin{array}{c}\overline{\mathbf{b}} \\ -1\end{array}\right)$ solves $A \mathbf{b}=\mathbf{0}$. If $\overline{\mathbf{b}}<\mathbf{0}$, then $\mathbf{z}:=-\mathbf{b}$ is a positive vector of the kernel of $A$. Whether $\overline{\mathbf{b}} \prec \mathbf{0}$ or not can be checked while using Cramer's rules to compute $\overline{\mathbf{b}}=\bar{A}^{-1} \overline{\mathbf{a}}$. If $\overline{\mathbf{b}} \prec \mathbf{0}, \mathbf{x}=\mathbf{z} /|\mathbf{z}|$ defines a vector in the one-simplex, solution to $A \mathbf{x}=\mathbf{0}$.

For instance the matrix $A=\left(\begin{array}{ccc}0 & a & b \\ -a & 0 & c \\ -b & -c & 0\end{array}\right)$ has the required property if $a c>0$ and $b c<0$ (or $a c>0$ and $a b<0$. Let $W=J /|\mathbf{z}|+\lambda^{-1} A$, where $\lambda>0$. There is a value $\lambda_{c}$ of $\lambda$ such that $W \succeq \mathbf{0}$ for $\lambda \geq \lambda_{c}$ and we have $W \mathbf{z}=\mathbf{1}$.

- The even case. Let $K$ be even. Suppose $A$ has rank $K-2$ so that 0 is a double eigenvalue of $A$. Then, up to a permutation of the rows and columns, $A$ can be written as:

$$
A=\left(\begin{array}{ccc}
\bar{A} & \overline{\mathbf{a}} & \overline{\mathbf{b}} \\
-\overline{\mathbf{a}}^{\prime} & 0 & -a_{K} \\
-\overline{\mathbf{b}}^{\prime} & a_{K} & 0
\end{array}\right),
$$

with $(i) \operatorname{rank}(\bar{A})=K-2$ and (ii) there exist $\overline{\mathbf{c}}$ and $\overline{\mathbf{d}}$ of dimension $K-2$ such that:

$$
\left(\begin{array}{c}
\bar{A} \\
-\overline{\mathbf{a}}^{\prime} \\
-\overline{\mathbf{b}}^{\prime}
\end{array}\right) \overline{\mathbf{c}}=\left(\begin{array}{c}
\overline{\mathbf{a}} \\
0 \\
a_{K}
\end{array}\right) \text { and }\left(\begin{array}{c}
\bar{A} \\
-\overline{\mathbf{a}}^{\prime} \\
-\overline{\mathbf{b}}^{\prime}
\end{array}\right) \overline{\mathbf{d}}=\left(\begin{array}{c}
\overline{\mathbf{b}} \\
-a_{K} \\
0
\end{array}\right) .
$$

Thus, $\bar{A} \overline{\mathbf{c}}=\overline{\mathbf{a}}$ defines uniquely $\overline{\mathbf{c}}), \overline{\mathbf{a}}^{\prime} \overline{\mathbf{c}}=0$ because $\bar{A}$ is skew-symmetric and $-\overline{\mathbf{b}}^{\prime} \overline{\mathbf{c}}=a_{K}$ fixing $a_{K}$ ). Similarly, $\bar{A} \overline{\mathbf{d}}=\overline{\mathbf{b}}$ defines uniquely $\overline{\mathbf{d}}, \overline{\mathbf{b}}^{\prime} \overline{\mathbf{d}}=0$ because $\bar{A}$ is skew-symmetric and $\overline{\mathbf{a}}^{\prime} \overline{\mathbf{d}}=-\overline{\mathbf{b}}^{\prime} \overline{\mathbf{c}}=a_{K}$.

Under these conditions, the vectors $\mathbf{c}=[\overline{\mathbf{c}},-1,0]^{\prime}$ and $\mathbf{d}=[\overline{\mathbf{d}}, 0,-1]^{\prime}$ obey $A \mathbf{c}=\mathbf{0}$ and $A \mathbf{d}=\mathbf{0}$; so they are the two linearly independent vectors of the kernel of $A$. If both $\overline{\mathbf{c}} \prec \mathbf{0}$ and $\overline{\mathbf{d}} \prec \mathbf{0}$, then $[-\overline{\mathbf{c}}, 1,0]^{\prime}$ and $[-\overline{\mathbf{d}}, 0,1]^{\prime}$ are two nonnegative linearly independent vectors of the kernel of $A$. Then $A$ can be written as:

$$
A=\left(\begin{array}{ccc}
\bar{A} & \overline{\mathbf{b}}_{1} & \overline{\mathbf{b}}_{2} \\
-\overline{\mathbf{b}}_{1}^{\prime} & 0 & 0 \\
-\overline{\mathbf{b}}_{2}^{\prime} & 0 & 0
\end{array}\right)
$$

The sub-matrix $\widetilde{A}:=\left(\begin{array}{cc}\bar{A} & \mathbf{b}_{1} \\ -\mathbf{b}_{1}^{\prime} & 0\end{array}\right)$ is amenable to the previous study with $K$ replaced by $K-1$; so it has rank $K-2$.

3.2. Asymmetric permutations. Let us note by $\circ$ the Hadamard product of matrices. Let $S$ be a permutation matrix defined by a permutation $\sigma$ that has no fixed points nor 2-cycles. Then $S \circ S^{\prime}=\mathbf{0}$ and $S$ is called a strictly asymmetric permutation matrix. Let $Q=\left(S-S^{\prime}\right) / 2$ and $\mathbf{z} \succ \mathbf{0}$. Define $A=D_{\mathbf{z}}^{-1} Q D_{\mathbf{z}}^{-1}$. We have $A^{\prime}=-A$ and, in view of $Q \mathbf{1}=\mathbf{0}$ and $\mathbf{1}^{\prime} Q=\mathbf{0}$, we find $A \mathbf{z}=D_{\mathbf{z}}^{-1} Q \mathbf{1}=\mathbf{0}$ and $\mathbf{z}^{\prime} A=\mathbf{1}^{\prime} Q D_{\mathbf{z}}^{-1}=\mathbf{0}$. 
Let $K$ be odd. Let us define the following sequence $\left(S_{l}: l=1, . .,(K-1) / 2\right)$ of strictly asymmetric permutation matrices. Let $S_{1}$ be one of these matrices, then the matrix $Q_{1}:=S_{1}-S_{1}^{\prime}$ has $2 K$ entries \pm 1 and $A_{1}:=D_{\mathbf{z}}^{-1} Q_{1} D_{\mathbf{z}}^{-1}$ has the desired properties. Now, for each $k=2, . .,(K-1) / 2$ we take a strictly asymmetric permutation matrix $S_{k}$ such that $S_{k} \circ\left(S_{k-1}+S_{k-1}^{\prime}\right)=\mathbf{0}$. Define $\bar{S}_{k}:=S_{k-1}+S_{k}$, so $\bar{S}_{k} \circ \bar{S}_{k}^{\prime}=\mathbf{0}$. Then $Q_{k}:=\bar{S}_{k}-\bar{S}_{k}^{\prime}$ has $2 k K$ entries \pm 1 and $A_{k}:=D_{\mathbf{z}}^{-1} Q_{k} D_{\mathbf{z}}^{-1}$ has the same properties as $A$. The matrix $Q_{(K-1) / 2}:=\bar{S}_{(K-1) / 2}-\bar{S}_{(K-1) / 2}^{\prime}$ has $K(K-1)$ entries \pm 1 and $A_{(K-1) / 2}:=D_{\mathbf{z}}^{-1} Q_{(K-1) / 2} D_{\mathbf{z}}^{-1}$ has the desired properties but it has no zero entries outside the diagonal. This shows how to generate skewsymmetric matrices $Q$ with entries $Q_{k, l} \in\{-1,1\}$ for all $k \neq l$, iteratively nesting 'mutually orthogonal' strictly asymmetric permutation matrices.

\section{Nonsymmetric Nonnegative Matrices sharing same Perron EIGENVECTOR}

Let $B$ be a nonnegative nonsymmetric irreducible matrix such that $B$ and $B^{\prime}$ share the same Perron eigenvector $\mathbf{z} \succ \mathbf{0}$, so with $B \mathbf{z}=B^{\prime} \mathbf{z}=\rho \mathbf{z}$, where $\rho>0$ is the real Perron eigenvalue (the spectral radius of $B$, see Horn and Johnson 1985). Then, for the skew-symmetric part $A:=\left(B-B^{\prime}\right) / 2$ of $B, A \mathbf{z}=\mathbf{0}$. For $\lambda>0$ define $W=J /|\mathbf{z}|+\lambda^{-1} A$. There is a value $\lambda_{c}$ such that $W \succeq 0$ for $\lambda \geq \lambda_{c}$ and we have $W \mathbf{z}=\mathbf{1}+\lambda^{-1} A \mathbf{z}=\mathbf{1}$.

Let $K$ be odd. We will construct a class of matrices $B$ satisfying the above properties by using a perturbation analysis of the symmetric case. Let $C \succeq 0$ be a symmetric irreducible nonnegative matrix and $\mathbf{z}=: \mathbf{z}_{0} \succ \mathbf{0}$ be the right and left Perron eigenvector associated to the Perron eigenvalue $\rho>0$. We can assume $\mathbf{z}_{0}^{\prime} \mathbf{z}_{0}=1$.

Let $\mathbf{z}_{i}, i=1, \ldots K-1$ be such that $\mathbf{z}_{i}, i=0, \ldots K-1$ constitute an orthonormal system, so $\mathbf{z}_{i}^{\prime} \mathbf{z}_{j}=\delta_{i, j}$. Let $D=\sum_{i=0}^{K-1} \gamma_{i} \mathbf{z}_{i} \mathbf{z}_{i}^{\prime}$, where $P_{i}=\mathbf{z}_{i} \mathbf{z}_{i}^{\prime}$ are projectors with $P_{i}^{2}=P_{i}$ and choose the real numbers $\gamma_{i}$ in such a way that $\gamma_{0}=1$ and $D \succeq 0$ (this is always possible because $D=\mathbf{z}_{0} \mathbf{z}_{0}^{\prime}+\sum_{i=1}^{K-1} \gamma_{i} \mathbf{z}_{i} \mathbf{z}_{i}^{\prime}$ and $\mathbf{z}_{0} \mathbf{z}_{0}^{\prime}$ is a matrix with strictly positive entries). Clearly $D$ is symmetric with $D^{2}=D D^{\prime}=\sum_{i=0}^{K-1} \gamma_{i}^{2} \mathbf{z}_{i} \mathbf{z}_{i}^{\prime}$. Then $B=C D$ is nonnegative and asymmetric because $[C, D]=B-B^{\prime} \neq 0$. Note also that $D \mathbf{z}_{0}=\mathbf{z}_{0}=D^{\prime} \mathbf{z}_{0}$ and $D \mathbf{z}_{i}=\gamma_{i} \mathbf{z}_{i} i=1, \ldots, K-1$, so that $\mathbf{z}_{i}$ (and $\mathbf{z}_{i}^{\prime}$ ) is the right (left) eigenvector of $D$ with eigenvalue $\gamma_{i}$.

We have $B \mathbf{z}=B \mathbf{z}_{0}=C D \mathbf{z}_{0}=C \mathbf{z}_{0}=\rho \mathbf{z}_{0}$ and $\mathbf{z}^{\prime} B=\mathbf{z}_{0}^{\prime} B=\mathbf{z}_{0}^{\prime} C D=\rho \mathbf{z}_{0}^{\prime} D=\rho \mathbf{z}_{0}^{\prime}$. So $B$ is an asymmetric nonnegative matrix with the same left and right Perron eigenvector $\mathbf{z}=: \mathbf{z}_{0} \succ \mathbf{0}$, associated to the Perron root $\rho$ of $C$.

4.1. Characterization in terms of stochastic matrices. Let $K>2$. It turns out that the class of $K \times K$ skew-symmetric matrices $A$ for which there is a unique $\mathbf{z} \succ \mathbf{0}$ such that $A \mathbf{z}=\mathbf{0}$ admits a representation in terms of some stochastic irreducible matrices. We note that the matrix $A$ has rank $K-1$ if $K$ is odd or $K-2$ if $K$ is even.

In the next result we shall consider $P$ a stochastic irreducible matrix. Then, it has a unique invariant probability vector $\pi$. We recall that $P$ obeys detailed balance if 
$\pi_{k} P_{k, l}=\pi_{l} P_{l, k}$ for all $k, l \in I_{K}$. This is equivalent to the fact that $P$ is self-adjoint with respect to the inner product defined by $\pi$.

Theorem 2. Let $K>2$.

(i) Let $P$ be a stochastic irreducible matrix not obeying detailed balance. Let $\boldsymbol{\pi}^{\prime}$ be its invariant row vector, so $\boldsymbol{\pi}^{\prime}=\boldsymbol{\pi}^{\prime} P$. Let $\mathbf{z} \succ \mathbf{0}$ and define $B=D_{\mathbf{z}}^{-1} D_{\boldsymbol{\pi}} P D_{\mathbf{z}}^{-1} \succeq \mathbf{0}$. Then $D_{\pi} P$ is an irreducible nonnegative matrix whose row and column sum vectors coincide. Furthermore, $B \mathbf{z}=B^{\prime} \mathbf{z}$ and $A=\left(B-B^{\prime}\right) / 2$ is skew-symmetric such that $A \mathbf{z}=\mathbf{0}$.

(ii) Any matrix $A$ with the latter property can be represented as $\left(B-B^{\prime}\right) / 2$, where $B=D_{\mathbf{z}}^{-1} D_{\boldsymbol{\pi}} P D_{\mathbf{z}}^{-1}$.

Proof: $(i) B \mathbf{z}=B^{\prime} \mathbf{z}=D_{\mathbf{z}}^{-1} \boldsymbol{\pi}$ and so $A \mathbf{z}=\mathbf{0}$. We have $\left(D_{\boldsymbol{\pi}} P\right) \mathbf{1}=\left(D_{\boldsymbol{\pi}} P\right)^{\prime} \mathbf{1}=\boldsymbol{\pi}$. When $P$ obey detailed balance, $\pi_{k} P_{k, l}=\pi_{l} P_{l, k}$ and so $B_{k, l}=B_{l, k}$ leading to a degenerate $A \equiv \mathbf{0}$. The one-dimensional simplex for which case $K=2$ is ruled out because in this case $P$ always obeys detailed balance.

(ii) Let $A$ be a skew-symmetric matrix such that $A \mathbf{z}=\mathbf{0}$ for some $\mathbf{z} \succ \mathbf{0}$. Assume $\operatorname{rank}(A)=K-1$, which entails that $K$ is odd. Let $\mathbf{w} \succ \mathbf{0}$ and $S=\left(\mathbf{w w}^{\prime}\right) / \mathbf{w}^{\prime} \mathbf{z}$ a rank 1 symmetric matrix. Let $B=S+A$ with $\operatorname{rank}(B)=K$. Then, $A=\left(B-B^{\prime}\right) / 2$, where $B$ is an irreducible nonnegative matrix such that $B \mathbf{z}=B^{\prime} \mathbf{z}=\mathbf{w}$. Further, $C:=D_{\mathbf{z}} B D_{\mathbf{z}} \succeq \mathbf{0}$ is an irreducible nonnegative matrix whose row and column sum vectors coincide with $C \mathbf{1}=D_{\mathbf{z}} B \mathbf{z}=C^{\prime} \mathbf{1}$. Let $\mathbf{c}=C \mathbf{1}=C^{\prime} \mathbf{1}$. Then $P=D_{\mathbf{c}}^{-1} C$ is an irreducible stochastic matrix with invariant measure $\boldsymbol{\pi}=\mathbf{c}$. Finally, one can choose $\mathbf{w}=D_{\mathbf{z}}^{-1} \boldsymbol{\pi}$, fully characterizing $B$. Because $B$ is full rank, so is $C$ and then also $P$.

As an example consider $P$ a bi-stochastic nonsymmetric matrix. The invariant vector is $\boldsymbol{\pi}^{\prime}=\mathbf{1}^{\prime}$ and so $D_{\boldsymbol{\pi}}$ is the identity. Therefore the representation of the above Theorem gives $A=D_{\mathbf{z}}^{-1} Q D_{\mathbf{z}}^{-1} \succeq 0$, where $Q=\left(P-P^{\prime}\right) / 2$.

Corollary 3. (i) If $\mathbf{z}=\boldsymbol{\pi}, B=P D_{\boldsymbol{\pi}}^{-1}$ and $B \mathbf{z}=B^{\prime} \mathbf{z}=\mathbf{1}$. Then $A=\left(B-B^{\prime}\right) / 2$ is skew-symmetric such that $A \mathbf{z}=\mathbf{0}$.

(ii) If $\mathbf{z}=D_{\boldsymbol{\pi}}^{1 / 2} \mathbf{1}, B=D_{\boldsymbol{\pi}}^{1 / 2} P D_{\boldsymbol{\pi}}^{-1 / 2}$ and $B \mathbf{z}=B^{\prime} \mathbf{z}=\mathbf{z}$ so $\mathbf{z}$ is the common left and right eigenvector of $B$ associated to the eigenvalue 1 . Then $A=\left(B-B^{\prime}\right) / 2$ is skew-symmetric such that $A \mathbf{z}=\mathbf{0}$.

(iii) Let $\rho>0$. Then $B=\rho D_{\boldsymbol{\pi}}^{1 / 2} P D_{\boldsymbol{\pi}}^{-1 / 2}$ is a nonnegative matrix with $\mathbf{z}$ as the common left and right Perron eigenvector associated to the eigenvalue $\rho$. Any matrix $B$ with this latter property can be represented in this way.

Proof: Only (iii) deserves a proof. Let $B \succeq \mathbf{0}$ be an irreducible nonnegative matrix sharing $\mathbf{z} \succ \mathbf{0}$ as a common left and right Perron eigenvector. Define $P=\rho^{-1} D_{\mathbf{z}}^{-1} B D_{\mathbf{z}}$. Then $P \mathbf{1}=\rho^{-1} D_{\mathbf{z}}^{-1} B \mathbf{z}=\mathbf{1}$ so $P$ is stochastic. We have $\boldsymbol{\pi}^{\prime}=\boldsymbol{\pi}^{\prime} P$, where $\boldsymbol{\pi}=D_{\mathbf{z}}^{2} \mathbf{1}$.

Let $K$ be even, $A=\left(B-B^{\prime}\right) / 2$ and $S=\left(B+B^{\prime}\right) / 2$. Assume $\operatorname{rank}(A)=K-2$. $\operatorname{Ker}(A)$ consists of two complex conjugate linearly independent and orthogonal eigenvectors $\mathbf{z}_{1}$ and $\mathbf{z}_{2}=\overline{\mathbf{z}}_{1}$ (with $\mathbf{z}_{1} \cdot \mathbf{z}_{2}:=\sum_{k} \mathbf{z}_{1, k} \overline{\mathbf{z}}_{2, k}=\overline{\mathbf{z}_{2} \cdot \mathbf{z}_{1}}$ ) and $\mathbf{z}=\xi \mathbf{z}_{1}+$ $\bar{\xi} \mathbf{z}_{2}=2 \operatorname{Re}\left(\xi \mathbf{z}_{1}\right) \succ \mathbf{0}$ for some complex number $\xi$ with complex conjugate $\bar{\xi}$. Then 
$B=S+A$ with $\operatorname{rank}(B)=K$ and $B \mathbf{z}=B^{\prime} \mathbf{z}=S \mathbf{z}=\mathbf{w}$. Note that when we take a realization of a random stochastic matrix $P$ obtained while considering that its rows are drawn independently from Dirichlet $(1, . ., 1)$ distributions. With probability 1 , $P \succ \mathbf{0}$ and it is irreducible with invariant measure $\boldsymbol{\pi}$. Let $B=D_{\mathbf{z}}^{-1} D_{\boldsymbol{\pi}} P D_{\mathbf{z}}^{-1}$; then 0 is a double eigenvalue of $A=\left(B-B^{\prime}\right) / 2$ with eigenvectors $\mathbf{z}_{1}$ and $\mathbf{z}_{2}=\overline{\mathbf{z}}_{1}$ generating $\operatorname{Ker}(A)$ in $\mathbb{C}$ and $\mathbf{z}$ can be chosen as $\mathbf{z}=\xi \mathbf{z}_{1}+\bar{\xi} \mathbf{z}_{2}=2 \operatorname{Re}\left(\xi \mathbf{z}_{1}\right) \succ \mathbf{0}$ for some $\xi$.

4.2. Stability of polymorphic states. Let $\Lambda$ be a nonsymmetric bi-substochastic matrix, that is $\Lambda \neq \Lambda^{\prime}, \Lambda \succeq 0$ with $\Lambda \mathbf{1}:=\mathbf{q}<\mathbf{1}$ and $\Lambda^{\prime} \mathbf{1}:=\mathbf{r}<\mathbf{1}$. Let $\lambda>0$. Then, the matrices $B=\lambda^{-1}(I-\Lambda)^{-1}$ and $B^{\prime}=\lambda^{-1}\left(I-\Lambda^{\prime}\right)^{-1}$ are potential matrices satisfying $B \neq B^{\prime}$. Denote by $\mathbf{z}$ and $\mathbf{v}$ the solutions of $B \mathbf{z}=\mathbf{1}$ and $B^{\prime} \mathbf{v}=\mathbf{1}$, which are nonnegative. Since $\mathbf{v}^{\prime} B \mathbf{z}=\mathbf{v}^{\prime} \mathbf{1}=\mathbf{1}^{\prime} \mathbf{z}$, we get $|\mathbf{z}|=|\mathbf{v}|$. Assume $\mathbf{q}=\mathbf{r}$. Then, $\mathbf{z}=\mathbf{v}$ and $\mathbf{z}=B^{-1} \mathbf{1}=\lambda(\mathbf{1}-\mathbf{q})$. Let $A:=\left(B-B^{\prime}\right) / 2$ and $\mathbf{z}=\lambda(\mathbf{1}-\mathbf{q})$. Clearly $A \mathbf{z}=\left(B \mathbf{z}-B^{\prime} \mathbf{z}\right) / 2=\mathbf{0}$ but also $A \mathbf{z}_{0}=\mathbf{0}$, where $\mathbf{z}_{0}:=\mathbf{1}-\mathbf{q}$.

Let $W:=\left|\mathbf{z}_{0}\right|^{-1} J+A$. It has nonnegative entries if and only if $\lambda \geq \lambda_{c}=$ $\left.\left(\left|\mathbf{z}_{0}\right| \max _{k, l}\left[(I-\Lambda)^{-1}\right]_{k, l}\right) / 2\right)$, it satisfies $W_{i, j}+W_{j, i}=2 /\left|\mathbf{z}_{0}\right|$ and $W$ has a positive right equilibrium potential obeying $W \mathbf{z}_{0}=\mathbf{1}$.

Consider the following dynamics over $S_{K}: \mathbf{p}(\mathbf{x})=D_{\mathbf{x}} W \mathbf{x} / \omega(\mathbf{x})$ with $\omega(\mathbf{x}):=$ $\mathbf{x}^{\prime} W \mathbf{x}$. Since $\mathbf{x}^{\prime} A \mathbf{x}=0$ we get $\mathbf{x}^{\prime} W \mathbf{x}=1 /\left|\mathbf{z}_{0}\right|$. This is also

$$
\mathbf{p}(\mathbf{x})=\left|\mathbf{z}_{0}\right| D_{\mathbf{x}} W \mathbf{x}=\left|\mathbf{z}_{0}\right| D_{W \mathbf{x}} \mathbf{x}=\mathbf{x}+\left|\mathbf{z}_{0}\right| D_{\mathbf{x}} A \mathbf{x} \text { or } \Delta \mathbf{x}=\left|\mathbf{z}_{0}\right| D_{\mathbf{x}} A \mathbf{x},
$$

Note that $\omega(\mathbf{x})=\mathbf{x}^{\prime} W \mathbf{x}$ is a constant of motion because $W$ is a flat fitness matrix. This dynamics has a polymorphic equilibrium state $\mathbf{x}_{e q}=\mathbf{z}_{0} /\left|\mathbf{z}_{0}\right|$. The stability condition of $\mathbf{x}_{e q}$ results from the study of the eigenvalues of the Jacobian matrix $J_{e q}:=\left[\frac{\partial \mathbf{p}(\mathbf{x})}{\partial \mathbf{x}}\right]_{\mathbf{x}_{e q}}$ at equilibrium, acting to the right on zero sum vectors $\boldsymbol{\delta}=\mathbf{x}-\mathbf{x}_{e q}$, namely

$$
J_{e q}=I+\frac{1}{\omega\left(\mathbf{x}_{e q}\right)} D_{\mathbf{x}_{e q}} W=I+D_{\mathbf{z}_{0}}\left(\frac{J}{\left|\mathbf{z}_{0}\right|}+A\right)=I+\mathbf{x}_{e q} \mathbf{1}^{\prime}+D_{\mathbf{z}_{0}} A .
$$

On the zero-sum eigenspace, $J_{e q}$ now reduces to $J_{e q}=I+D_{\mathbf{z}_{0}} A$. The equilibrium state $\mathbf{x}_{e q}$ is stable if the spectral radius of $J_{e q}$ is $<1$ and unstable otherwise. Since the diagonal terms of $A$ vanish, the centers of the Gerschgorin disks of $J_{e q}$ are located at point $(1,0)$ of the complex plane, so $\rho\left(J_{e q}\right)>1$ and $\mathbf{x}_{e q}$ is unstable (a result due to Liberman 1990).

4.3. GUM matrices. We will introduce GUM matrices and show that the class of GUM nonsymmetric matrices $W$ for which the equilibrium potential vectors of $W$ and $W^{\prime}$ are strictly positive and coincide, are necessarily symmetric. Hence, this class of potential matrices do not admit nontrivial skew-symmetric matrices supplied by Theorem 2 .

For the definition and main properties of GUM matrices see (McDonald et al. 1995, Nabben and Varga 1995 and Dellacherie et al. 2000). Here we give the GUM matrix in its nested block form (NBF) matrices rather than in terms of inequalities as in the case of ultrametric matrices, but such a characterization exists. The matrix 
$W=\left(W_{i, j}: i, j \in I_{K}\right)$ is a GUM matrix in its NBF if there exists a non-trivial partition of the index set $I_{K}=I^{1} \cup I^{2}$, such that

$$
W=\left(\begin{array}{cc}
W^{1} & \alpha_{W}, \mathbf{1}_{I^{1}} \mathbf{1}_{I^{2}}^{\prime} \\
\beta_{W} \mathbf{1}_{I^{2}} \mathbf{1}_{I^{1}}^{\prime} & W^{2}
\end{array}\right)
$$

with

$$
\alpha_{W}=\min _{i, j \in I_{K}} W_{i j} \text { and } \beta_{W} \leq \min \left\{\min _{i, j \in I^{1}, i \geq j} W_{i j}, \min _{i, j \in I^{2}, i \geq j} W_{i, j}\right\} ;
$$

and such that $W^{1}$ and $W^{2}$ admit also this representation in NBF. This algorithmic way of defining a nested block form matrix generates a family of nested block form submatrices, we simple call it a family of nested block form matrices defining $W$.

The GUM matrix $W$ is nonsingular if no row and no column is equal to 0 , and no two rows nor two columns are equal. We will assume that this is the case. On the other hand it was shown in (McDonald et al. 1995 and Nabben and Varga 1995) that a nonsingular GUM matrix $W$ is a potential matrix, so it has right and left nonnegative equilibrium potential vectors denoted respectively by $\mathbf{z}$ and $\mathbf{v}$.

Let us mention that a GUM matrix is symmetric if and only if it is ultrametric. So, for dimension 1 a nonsingular GUM matrix is ultrametric and $\mathbf{z}=\mathbf{v}=\alpha_{W}^{-1}$.

If $W$ is a GUM matrix has a constant row and a constant column (we can assume that it is the first row and the first column), both with constant value $\alpha_{W}$ then $\mathbf{z}=\mathbf{v}=\alpha_{W}^{-1} e_{1}$, where $e_{1}$ is the vector with a 1 in coordinate 1 and all the other coordinates are 0 . Moreover in the decomposition (16), we have that $W^{1}$ is a symmetric matrix whose off-diagonal matrix is the constant $\alpha_{W}$; we also have $\beta_{W}=\alpha_{W}$ and $W^{2}$ is a GUM matrix (necessarily with $\beta_{W^{2}} \geq \beta_{W}$ and $\alpha_{W^{2}} \geq \alpha_{W}$ ).

Hence, a necessary and sufficient condition in order that the right and left $\mathbf{z}$ and $\mathbf{v}$ equilibrium potential vectors are (strictly) positive is that every matrix belonging to a family of nested block form matrices defining $W$ has no constant row and constant column equal to its minimum value.

Theorem 4. Let $W$ be a nonsingular GUM matrix. Then, if $\mathbf{z} \succ \mathbf{0}$ and $\mathbf{v} \succ \mathbf{0}$ we have $\mathbf{z}=\mathbf{v}$ if and only if $W$ is symmetric (so $W$ is an ultrametric matrix).

Proof: Assume $\mathbf{z}=\mathbf{v}$, we must show $W$ is symmetric. Let us make the partition

$$
\mathbf{z}=\left(\begin{array}{l}
\mathbf{z}_{1} \\
\mathbf{z}_{2}
\end{array}\right)
$$

with $\mathbf{z}_{1}$ of dimension $\left|I^{1}\right|$ and $\mathbf{z}_{2}$ of dimension $\left|I^{2}\right|$. Denote $\alpha=\alpha_{W}$ and $\beta=\beta_{W}$. We have

$$
W \mathbf{z}=\left(\begin{array}{c}
W^{1} \mathbf{z}_{1}+\alpha\left|\mathbf{z}_{2}\right| \mathbf{1}_{I^{1}} \\
\beta\left|\mathbf{z}_{1}\right| \mathbf{1}_{I^{1}}+W^{2} \mathbf{z}_{2}
\end{array}\right)
$$

and

$$
\mathbf{z}^{\prime} W=\left(\mathbf{z}_{1}^{\prime} W^{1}+\beta\left|\mathbf{z}_{2}\right| \mathbf{1}_{I^{1}}^{\prime}, \alpha\left|\mathbf{z}_{1}\right| \mathbf{1}_{I^{2}}^{\prime}+\mathbf{z}_{2}^{\prime} W^{2}\right) .
$$

From $W \mathbf{z}=\mathbf{1}$ and $\mathbf{z}^{\prime} W=\mathbf{1}^{\prime}$ we find

$$
\begin{aligned}
& W^{1} \mathbf{z}_{1}=\left(1-\alpha\left|\mathbf{z}_{2}\right|\right) \mathbf{1}_{I^{1}}, W^{2} \mathbf{z}_{2}=\left(1-\beta\left|\mathbf{z}_{1}\right|\right) \mathbf{1}_{I^{1}} \\
& \mathbf{z}_{1}^{\prime} W^{1}=\left(1-\beta\left|\mathbf{z}_{2}\right|\right) \mathbf{1}_{I^{1}}^{\prime}, \mathbf{z}_{2}^{\prime} W^{2}=\left(1-\alpha\left|\mathbf{z}_{1}\right|\right) \mathbf{1}_{I^{2}}^{\prime} .
\end{aligned}
$$


Therefore

$$
\begin{aligned}
& \mathbf{z}_{1}=\left(1-\alpha\left|\mathbf{z}_{2}\right|\right) \mathbf{z}_{W^{1}}, \quad \mathbf{z}_{2}=\left(1-\beta\left|\mathbf{z}_{1}\right|\right) \mathbf{z}_{W^{2}} \\
& \mathbf{z}_{1}=\left(1-\beta\left|\mathbf{z}_{2}\right|\right) \mathbf{z}_{W^{1}}, \mathbf{z}_{2}=\left(1-\alpha\left|\mathbf{z}_{1}\right|\right) \mathbf{z}_{W^{2}} .
\end{aligned}
$$

Then, from $\alpha \leq \beta$ we get $\alpha=\beta$. We also deduce

$$
\mathbf{z}_{W^{1}}=\mathbf{v}_{W^{1}} \text { and } \mathbf{z}_{W^{2}}=\mathbf{v}_{W^{2}} .
$$

Moreover, $\mathbf{z}_{1} \succ \mathbf{0}$ and $\mathbf{z}_{2} \succ \mathbf{0}$ also implies $\mathbf{z}_{W^{1}}=\mathbf{v}_{W^{1}} \succ \mathbf{0}$ and $\mathbf{z}_{W^{2}}=\mathbf{v}_{W^{2}} \succ \mathbf{0}$. A recurrence argument gives $\alpha_{W^{k}}=\beta_{W^{k}}$ for every matrix $W^{k}$ belonging to a family of nested block form matrices defining $W$; so $W$ is symmetric.

We note that we can make a perturbation of a strictly ultrametric matrix to get a nonsymmetric potential matrix such that it shares its Perron eigenvector with its transpose. But the above result implies that this matrix is not a GUM matrix.

\section{Acknowledgments:}

T. Huillet and S. Martínez acknowledge support from the Project Basal PFB 03 of the CONICYT, Chile. S. Martínez thanks the University of Cergy-Pontoise for a visiting Professor opportunity. T. Huillet also acknowledges support from the "Chaire Modélisation mathématique et biodiversité " and from the labex MME-DII

Center of Excellence (Modèles mathématiques et économiques de la dynamique, de l'incertitude et des interactions, ANR-11-LABX-0023-01 project).

\section{REFERENCES}

[1] Atkinson FV, Watterson GA, Moran PAP (1960) A matrix inequality. Quart. J. Math. Oxford Ser. 11, 137-140.

[2] Bapat RB, Raghavan TES (1997) Nonnegative Matrices and Applications. Cambridge University Press, vol 64 .

[3] Bürger R (2000) The mathematical theory of selection, recombination, and mutation. Wiley Series in Mathematical and Computational Biology. John Wiley \& Sons, Ltd., Chichester.

[4] Castilloux AM, Lessard S (1995) The fundamental theorem of natural selection in Ewens' sense (case of many loci). Theor. Popul. Biol. 48(3), 306-315.

[5] Dellacherie C, Martínez S, San Martín J (2000) Description of the sub-Markov kernel associated to generalized ultrametric matrices. An algorithmic approach. Linear Algebra Appl. 318 , No. 1-3, 1-21.

[6] Ewens WJ (2004) Mathematical population genetics. I.Theoretical introduction. Second edition. Interdisciplinary Applied Mathematics, 27. Springer-Verlag, New York.

[7] Ganikhodzhaev R, Mukhamedov F, Rozikov U (2011) Quadratic stochastic operators and processes: results and open problems. Infin. Dimens. Anal. Quantum. Probab. Relat. Top., $14(2), 279-$.

[8] Hofbauer J, Sigmund K (1998) Evolutionary games and population dynamics. Cambridge University Press, Cambridge.

[9] Horn RA, Johnson CR (1985) Matrix analysis. Cambridge University Press, Cambridge.

[10] Karlin S (1984) Mathematical models, problems, and controversies of evolutionary theory. Bull. Amer. Math. Soc. (N.S.) 10(2), 221-274.

[11] Kimura M (1954) Process leading to quasi-fixation of genes in natural populations due to random fluctuation of selection intensities. Genetics 39, 280-295.

[12] Kingman JFC (1961a) A mathematical problem in population genetics. Proc. Cambridge Philos. Soc. $57,574-582$.

[13] Kingman JFC (1961b) On an inequality in partial averages. Quart. J. Math. Oxford Ser. 12, $78-80$. 
[14] Kingman JFC (1980) Mathematics of genetic diversity. CBMS-NSF Regional Conference Series in Applied Mathematics, 34. Society for Industrial and Applied Mathematics (SIAM), Philadelphia, Pa.

[15] Lessard S, Karlin S (1982) A criterion for stability-instability at fixation states involving an eigenvalue one with applications in population genetics, Theor. Popul. Biol., 22, 108-126.

[16] Lewontin RC, Ginzburg LR, Tuljapurkar SD (1978) Heterosis as an explanation for large amounts of genic polymorphism. Genetics, 88, 149-170.

[17] Liberman U (1990) Mendelian segregation: a choice between 'order' and 'chaos', J. Math. Biol. 28, 435-449.

[18] Liberman U (1991) On the relation between the instability of ESS in discrete dynamics and segregation distortion, J. Theor. Biol. 150, 421-436.

[19] Lyubich YI, Maistrovskii GD, Olkhovskii YG (1980) Selection-Induced Convergence to Equilibrium in a Single-Locus Autosomal Population, Probl. Peredachi Inf., Volume 16, Issue 1, 93-104.

[20] Mandel SPH (1959) The stability of a multiple allelic system. Heredity, 13, 289-302.

[21] Martínez S, Michon G, San Martín J (1994) Inverse of strictly ultrametric matrices are of Stieltjes type. SIAM J. Matrix Anal. Appl., 15, 98-106.

[22] McDonald JJ, Neumann M, Schneider H, Tsatsomeros M (1995) Inverse M-Matrix Inequalities and Generalized Ultrametric Matrices. Linear Algebra and its Applications, 220, 321-341.

[23] Nabben R, Varga RS (1994) A linear algebra proof that the inverse of a strictly ultrametric matrix is a strictly diagonally dominant Stieltjes matrix. SIAM J. Matrix Anal. Appl., 15(1), $107-113$.

[24] Nabben R, Varga RS (1995) Generalized ultrametric matrices - a class of inverse M-matrices. Linear Algebra and its Applications 220, 365-390.

[25] Nagylaki T (1992) Introduction to theoretical population genetics. Biomathematics, 21. Springer-Verlag, Berlin.

[26] Okasha S (2008) Fisher's fundamental theorem of natural selection - a philosophical analysis. The British Journal for the Philosophy of Science, 59(3), 319-351.

[27] Price GR (1972) Fisher's fundamental theorem made clear. Ann. Hum. Genet., Lond., 36(2), 129-140.

[28] Sanyal DC, Sarkar B (2013) Interaction between selection and segregation ratios with redefined concept of fitness differentials. TWMS J. App. Eng. Math. V.3, N.1, 87-102.

[29] Shashahani S (1979) A new mathematical framework for the study of linkage and selection. Mem. Amer. Math. Soc., 17.

[30] Tallis GM (1966) Equilibria under selection for $k$ alleles. Biometrics 22, 121-127.

[31] Weissing FJ, van Boven M (2001) Selection and segregation distortion in a sex-differentiated population. Theor. Popul. Biol., 60(4), 327-341.

1 Laboratoire de Physique Théorique et Modélisation, CNRS-UMR 8089 et UniverSité de Cergy-Pontoise, 2 Avenue Adolphe Chauvin, F-95302, Cergy-Pontoise, France, 2 Departamento de Ingeniería Matemática, Centro Modelamiento Matemático, Umi 2807, UChile-CNRS, Casilla 170-3 Correo 3, Santiago, Chile, ${ }^{3}$ Mathematisches Institut, Eberhard Karls Universität Tübingen, Auf der Morgenstelle 10,72076 Tübingen, Germany, Thierry.Huillet@U-Cergy.Fr, Smartine@Dim.UChile.Cl and martin.moehle@uniTUEBINGEN.DE. 